\title{
Diverse Protoplanetary Disk Morphology Produced by a Jupiter-mass Planet
}

\author{
Jaehan Bae ${ }^{1}$ (1), Paola Pinilla ${ }^{2}$ (1) , and Tilman Birnstiel ${ }^{3}$ (1) \\ ${ }^{1}$ Department of Terrestrial Magnetism, Carnegie Institution for Science, 5241 Broad Branch Road, NW, Washington, DC 20015, USA; jbae@carnegiescience.edu \\ ${ }_{3}^{2}$ Department of Astronomy/Steward Observatory, The University of Arizona, 933 North Cherry Avenue, Tucson, AZ 85721, USA \\ ${ }^{3}$ University Observatory, Faculty of Physics, Ludwig-Maximilians-Universität München, Scheinerstr. 1, D-81679 Munich, Germany \\ Received 2018 July 9; revised 2018 August 27; accepted 2018 August 28; published 2018 September 6
}

\begin{abstract}
Combining hydrodynamic planet-disk interaction simulations with dust evolution models, we show that protoplanetary disks with a giant planet can reveal diverse morphology in (sub)millimeter continuum, including a full disk without significant radial structure, a transition disk with an inner cavity, a disk with a single gap and a central continuum peak, and a disk with multiple rings and gaps. Such diversity originates from (1) the level of viscous transport in the disk, which determines the number of gaps a planet can open; (2) the size and spatial distributions of grains determined by the coagulation, fragmentation, and radial drift, which in turn affects the emissivity of the disk at (sub)millimeter wavelengths; and (3) the angular resolution used to observe the disk. In particular, our results show that disks with the same underlying gas distribution can have very different grain size/ spatial distributions and thus appearance in continuum, depending on the interplay among coagulation, fragmentation, and radial drift. This suggests that proper treatments for the grain growth have to be included in models of protoplanetary disks concerning continuum properties and that complementary molecular line observations are highly desired in addition to continuum observations to reveal the true nature of disks. The fact that a single planet can produce diverse disk morphology emphasizes the need to search for more direct, localized signatures of planets in order to confirm (or dispute) the planetary origin of observed ringed substructures.
\end{abstract}

Key words: hydrodynamics - planet-disk interactions - protoplanetary disks

\section{Introduction}

Followed by the revolutionary discovery of sets of rings and gaps in the millimeter continuum emission of the HL Tau disk (ALMA Partnership et al. 2015), concentric rings and gaps have been imaged in more than a dozen protoplanetary disks by now thanks to the Atacama Large Millimetre Array and optical/infrared telescopes equipped with adaptive optics. Such ringed substructures are found in disks around stars with a broad range of masses from subsolar masses (e.g., TW Hya; Andrews et al. 2016; Tsukagoshi et al. 2016) to $\sim 2$ solar masses (e.g., HD 163296; Isella et al. 2016), but also with various ages spanning from less than 1 million years old (e.g., GY 91; Sheehan \& Eisner 2018) to nearly 10 million years old (e.g., TW Hya; Andrews et al. 2016; Tsukagoshi et al. 2016). Furthermore, ringed substructures have been observed using different techniques: (sub)millimeter/centimeter continuum (e.g., ALMA Partnership et al. 2015), molecular line emission (Teague et al. 2018), and optical/infrared scattered light (e.g., Avenhaus et al. 2018). These observations thus seem to suggest that ringed substructures are pervasive on scales of 0 !" 1 ( $\simeq 10-20$ au in linear scale) in protoplanetary disks (Zhang et al. 2016; Avenhaus et al. 2018).

The origin of observed rings and gaps is unfortunately still far from clear. The interaction between planet and disk (Lin \& Papaloizou 1980) is certainly an intriguing possibility but other processes including various types of fluid instabilities (Takahashi \& Inutsuka 2014; Flock et al. 2017; Dullemond \& Penzlin 2018), dust property changes across condensation fronts (Zhang et al. 2015; Okuzumi et al. 2016), and radial variation of magnetic activities (Johansen et al. 2009) can also produce similar ringed substructures, and we do not yet have a conclusive way to differentiate these mechanisms based on observed features.
The situation became more complicated because it is shown that one planet can open multiple gaps (Bae et al. 2017; see also Dong et al. 2017). Planets excite multiple spiral arms (Bae \& Zhu 2018a) and each spiral arm can open a gap at the radial location in which it shocks the disk gas (Bae et al. 2017). In such a case, even with arbitrarily powerful observing facilities, we would not detect a planet in the planet-induced gaps other than the primary one, paradoxically.

Assuming that an observed gap is created by a planet orbiting within them, one can estimate the planet's mass using hydrodynamic simulations or empirical relations between planet mass and gap depth/width (e.g., Kanagawa et al. 2015). Figure 1 presents such attempts compiled from the literature, in which we plot the mass and semimajor axis of hypothesized planets required to reproduce the observed gaps. The planet masses obtained by both approaches (i.e., simulations, empirical relations) depend on the physical properties of the underlying disk, including the disk aspect ratio and the level of viscous transport, but it is interesting to note that the estimated masses are broadly consistent for the 12 disks presented in the figure (19 gaps in total). The estimated masses range from about a few percent of a Jupiter-mass to one Jupiter-mass, coincident with the masses of solar system's gas/ice giants. If (and only if) confirmed, these gap-opening protoplanets will provide us critical insights into studies of planet formation. Also, depending on their future migration and accretion, we may be witnessing planets that will eventually be miniNeptune-mass planets or hot/warm Jupiters, for which we now have a decent number of confirmed populations in mature planetary systems. As an aside, it is also worth mentioning that the planet masses assumed to reproduce observed gaps are approximately an order of magnitude smaller than the ones needed to reproduce observed spiral arms, presumably because the spiral arms driven by (sub-)Jovian-mass planets are too 

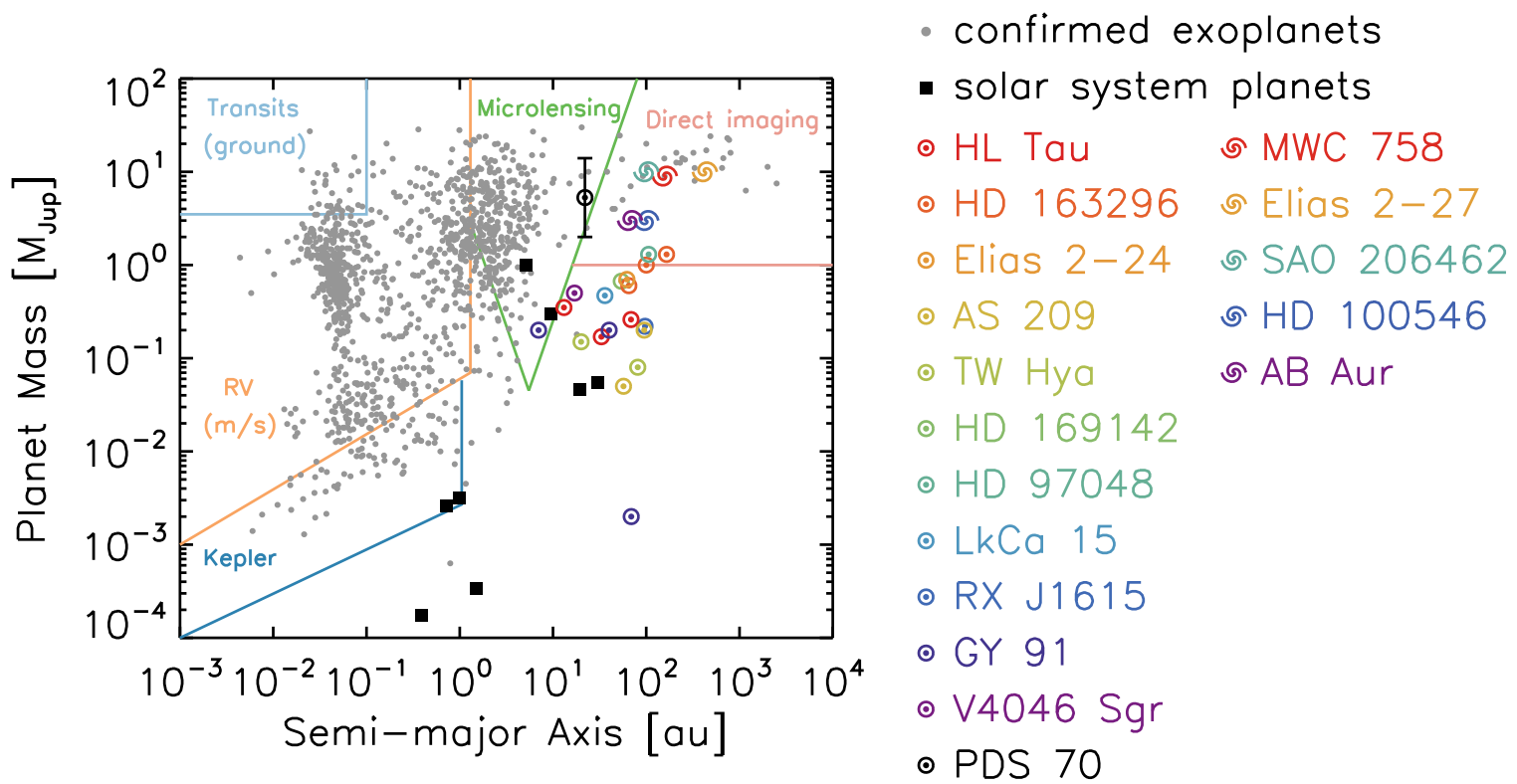

Figure 1. Semimajor axis and mass of hypothesized planets assumed to reproduce the observed gaps in protoplanetary disks $(\odot$ symbols), compiled from the literature: HL Tau (Jin et al. 2016), HD 163296 (Teague et al. 2018), Elias 2-24 (Dipierro et al. 2018), AS 209 (Fedele et al. 2018), TW Hya, HD 169142, HD 97048, LkCa 15, RX J1615 (Dong \& Fung 2017a), GY 91 (Sheehan \& Eisner 2018), and V4046 Sgr (Ruíz-Rodríguez et al. 2018, in preparation). The predictions are made using either planet-disk interaction simulations (HL Tau, HD 163296, Elias 2-24, AS 209, and V4046 Sgr) or empirical relations between the planet's mass and gap width (TW Hya, HD 169142, HD 97048, LkCa 15, RX J1615, and GY 91). For the estimations collected from Dong \& Fung (2017a), we adopted the masses obtained with a disk viscosity of $\alpha=10^{-3}$. Using a factor of 10 larger/smaller disk viscosity would result in about a factor of 3 larger/smaller planet masses (Dong \& Fung 2017a). Also shown with spiral symbols are the semimajor axis and mass of hypothesized planets needed to reproduce observed spiral arms, based on planet-disk interaction simulations: MWC 758 (Dong et al. 2015), Elias 2-27 (Meru et al. 2017), SAO 206462 (Bae et al. 2016 ), HD 100546 (Follette et al. 2017), AB Aur (Dong et al. 2016). In case of AB Aur, both spiral arms and gap are observed (Hashimoto et al. 2011, Tang et al. 2017). Note that some of the disks have directly imaged, confirmed/candidate companions: HD 100546 (Quanz et al. 2013, Currie et al. 2015), LkCa 15 (Sallum et al. 2015), and PDS 70 (Keppler et al. 2018). The error bar on PDS 70 represents the suggested mass range from (Keppler et al. 2018). The gray circles represent confirmed exoplanets as of 2018 May (https://exoplanetarchive.ipac.caltech.edu/). The black squares represent the eight solar system planets. The overplotted lines with color show illustrative estimates of the regions for which various exoplanet detection techniques have discovered exoplanets, similar to the shaded regions in Figure 6 of Gaudi (2012).

tightly wound and/or do not produce sufficient perturbations (Dong \& Fung 2017b; Bae \& Zhu 2018b).

In this paper, as a step forward to better understand the origin of observed ringed substructures, we examine the morphology of protoplanetary disks in millimeter continuum produced by a Jupiter-mass planet. Since we are concerned with millimeter continuum, we consider grain evolution-both spatial and size -in response to the gas structure that a Jupiter-mass planet creates. As we will show, depending on the physical properties of the disk and the angular resolution used for observations, a single giant planet can produce a diverse disk morphology in millimeter continuum emission: (1) a full disk without significant radial structure; (2) a transition disk with an inner cavity; (3) a disk with a single gap with a central continuum peak; and (4) a disk with multiple rings and gaps. From the perspective of differentiating possible gap-opening mechanisms, the diverse morphology a planet can produce emphasizes the need to search for more direct evidence of planets to confirm (or dispute) the planetary origin of observed ringed substructures.

This paper is organized as follows. We introduce our hydrodynamic and dust evolutionary models in Section 2 . We present simulation results in Section 3 and synthesized images of disks' continuum emission in Section 4. We summarize and present an outlook for future studies in Section 5.

\section{Methods and Background}

\subsection{Gas Evolution}

We run two-dimensional hydrodynamic calculations using FARGO3D (Masset 2000; Benítez-Llambay \& Masset 2016) to simulate the gas evolution. The initial disk assumes power-law gas surface density and temperature distributions following $\Sigma_{\text {gas }}(r)=$ $\Sigma_{\text {gas }, p}\left(r / r_{p}\right)^{-1}$ and $T(r)=T_{p}\left(r / r_{p}\right)^{-1 / 2}$, where $\Sigma_{\text {gas }, p}$ and $T_{p}$ are the gas surface density and temperature at the location of the planet $r=r_{p}$. The simulation domain extends from 2 to $200 \mathrm{au}$ in radius and from 0 to $2 \pi$ in azimuth. We adopt 2048 logarithmically spaced grid cells in the radial direction and 2792 uniformly spaced grid cells in the azimuthal direction. We include a $1 M_{\text {Jup }}$ planet orbiting around a $1 M_{\odot}$ star at $r_{p}=20 \mathrm{au}$. We assume a total disk mass of $0.02 M_{\odot}$ and a disk aspect ratio of 0.1 at $r=r_{p}$, with which $\Sigma_{\text {gas }, p}=7.1 \mathrm{~g} \mathrm{~cm}^{-2}$ and $T_{p}=127 \mathrm{~K}$, respectively. We add a viscosity $\alpha=5 \times 10^{-5}$ or $5 \times 10^{-4}$ to the gas (see Section 2.3), where $\alpha$ denotes a Shakura-Sunyaev viscosity parameter (Shakura \& Sunyaev 1973).

\subsection{Grain Evolution}

For the spatial and size evolution of grains, we adopt the model introduced in Birnstiel et al. (2010) which we briefly summarize here. In this model, grains evolve in response to the underlying gas structure, considering growth via coagulation, fragmentation due to turbulence, and radial drift. In practice, the grain evolution is simulated by solving the continuity equation for each radial grid cell and for each grain size bin. We adopt the azimuthally averaged gas surface density distribution from hydrodynamic simulations obtained after 1000 planetary orbital times as the initial condition, by which time the disk has reached a quasisteady state. The initial grain surface density is constructed assuming a uniform gas-to-dust ratio of 100 throughout the disk, and we add the entire solid mass $\left(0.0002 M_{\odot}\right)$ in $1 \mu \mathrm{m}$ grains. We 

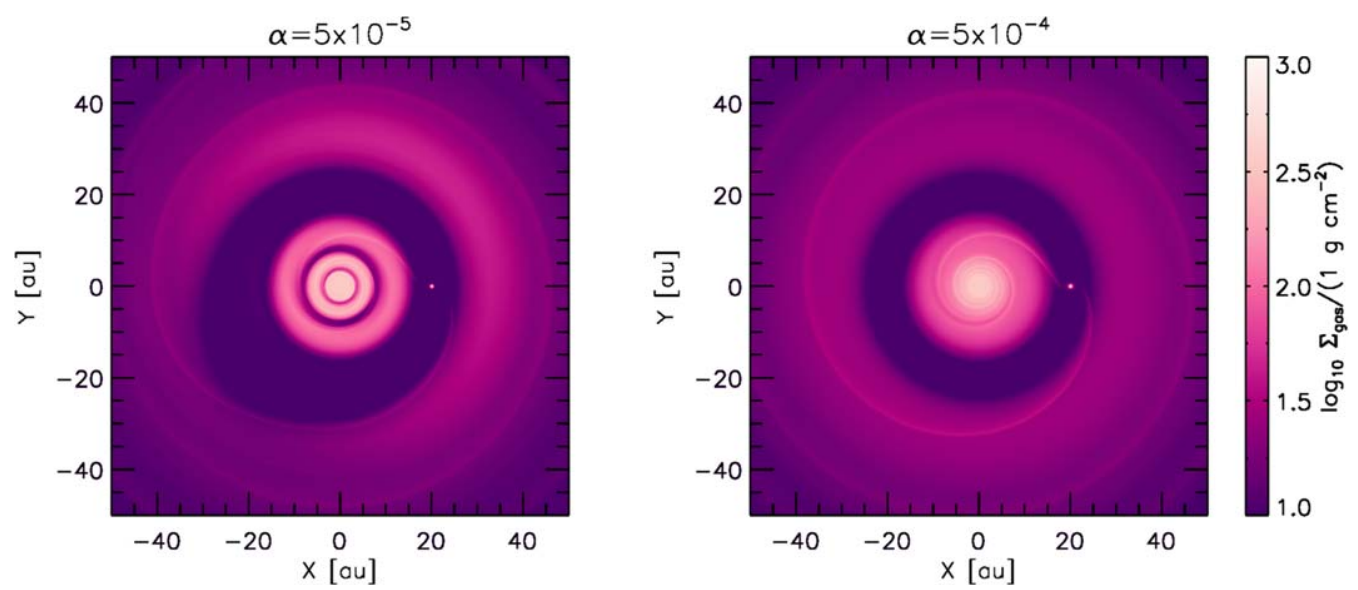

Figure 2. Gas surface density distributions from hydrodymanic simulations using (left) $\alpha=5 \times 10^{-5}$ and (right) $\alpha=5 \times 10^{-4}$. The Jupiter-mass planet (located at $X=20$ au and $Y=0$ au in the plot) opens three gaps in the low-viscosity disk, while it opens only one gap in the large-viscosity disk. See Figure 5 for azimuthally averaged profiles of the gas surface density.

use 180 logarithmically spaced bins for grain sizes, ranging from $1 \mu \mathrm{m}$ to $2 \mathrm{~m}$. The grain evolution model does not include the back reaction of grains onto the gas.

Grains grow via coagulation but their growth can be limited by two barriers. When the timescale for the fragmentation is shorter than the growth timescale, grains fragment into smaller bodies rather than growing further: the fragmentation barrier. Similarly, when the timescale for the radial drift at a certain location is shorter than the growth timescale, the further growth of grains at that location can be limited: the drift barrier.

While the chain of continuity equations has to be numerically solved to obtain the complete grain size distribution, the maximum size grains that can grow under the two barriers can be estimated by comparing the aforementioned timescales (e.g., Birnstiel et al. 2012). In a fragmentation-dominated regime, the maximum size that grains can grow $\left(a_{\mathrm{frag}}\right)$ is given by

$$
a_{\text {frag }} \simeq \frac{\sum_{\mathrm{gas}}}{\pi \rho_{s}} \frac{1-\sqrt{1-4 \Lambda_{\mathrm{frag}}^{2}}}{\Lambda_{\text {frag }}},
$$

where

$$
\Lambda_{\text {frag }} \equiv \frac{1}{3 \alpha_{\text {turb }}}\left(\frac{v_{\text {frag }}}{c_{s}}\right)^{2}
$$

$\rho_{s}$ is the bulk density of grains, $\alpha_{\text {turb }}$ is a parameter characterizing the level of turbulence (defined in a similar manner to the gas viscosity parameter $\alpha$ above), $v_{\text {frag }}$ is the fragmentation velocity of grains, and $c_{s}$ is the sound speed. In this work, we use $\rho_{s}=$ $1.2 \mathrm{~g} \mathrm{~cm}^{-2}$ and $v_{\text {frag }}=10 \mathrm{~m} \mathrm{~s}^{-1}$. In a drift-dominated regime, the maximum size that grains can grow $\left(a_{\text {drift }}\right)$ is given by

$$
a_{\mathrm{drift}} \simeq \frac{\Sigma_{\mathrm{gas}}}{\pi \rho_{s}} \frac{1-\sqrt{1-4 \Lambda_{\mathrm{drift}}^{2}}}{\Lambda_{\mathrm{drift}}},
$$

where

$$
\Lambda_{\text {drift }} \equiv \frac{\Sigma_{\text {grain }}}{\Sigma_{\text {gas }}}\left(\frac{v_{\text {Kep }}}{c_{s}}\right)^{2}\left|\frac{d \log P}{d \log r}\right|^{-1},
$$

$\Sigma_{\text {grain }}$ is the grain surface density, $v_{\text {Kep }}$ is the Keplerian velocity, and $P$ is the gas pressure. Note that the formulae given in Equations (1) and (3) are more general than the ones in the literature (cf., Birnstiel et al. 2015) to consider small grains having Stokes numbers of $S t \ll 1$ but also large grains having $S t \gtrsim 1$ (see also the discussions in, e.g., Birnstiel et al. 2010; Pinilla et al. 2012).

As can be inferred from Equations (1) and (3), for given disk density and temperature profiles, the level of turbulence $\left(\alpha_{\text {turb }}\right)$ determines whether the grain growth is limited by fragmentation or radial drift. In general, in a disk with strong turbulence, the grain growth is limited by fragmentation, whereas in a disk with weak turbulence fragmentation is inefficient and the grain growth is limited by radial drift. As we will show, however, there exist certain circumstances under which neither fragmentation nor radial drift could limit the growth of grains (see Model 1 below).

\subsection{Model Description}

We consider three models. Our fiducial model (hereafter Model 1) uses $\alpha=5 \times 10^{-5}$ in the hydrodynamic simulation and $\alpha_{\text {turb }}=5 \times 10^{-5}$ in the grain evolution calculation.

The second model (hereafter Model 2) uses $\alpha=5 \times 10^{-5}$ in the hydrodynamic simulation but an enhanced turbulence parameter $\alpha_{\text {turb }}=10^{-3}$ in the grain evolution calculation. The physical motivation for separating $\alpha$ and $\alpha_{\text {turb }}$ is the following. In the grain evolution calculation $\alpha_{\text {turb }}$ characterizes relative velocities between grains induced by turbulent gas motions, which in turn sets the maximum grain size due to fragmentation. On the other hand, in the hydrodynamic simulation $\alpha$ characterizes the efficiency of viscous transport of gas via any relevant physical process. The two parameters therefore do not necessarily characterize the same physical process. By purposely separating $\alpha_{\text {turb }}$ from $\alpha$, we examine how enhanced fragmentation alone can alter the appearance of the disk with a fixed gas structure, by changing the grain size distribution.

The third model (hereafter Model 3) assumes $\alpha=5 \times 10^{-4}$ in the hydrodynamic simulation and $\alpha_{\text {turb }}=5 \times 10^{-4}$ in the grain evolution calculation. The purpose of this model is to examine the number of rings and gaps created in a disk with stronger viscous transport.

\section{Simulation Results}

Figure 2 presents two-dimensional gas density distributions from hydrodynamic simulations. When $\alpha=5 \times 10^{-5}$ is assumed, the Jupiter-mass planet opens three gaps in the disk. The planet opens the primary gap around its orbit at $20 \mathrm{au}$. In 


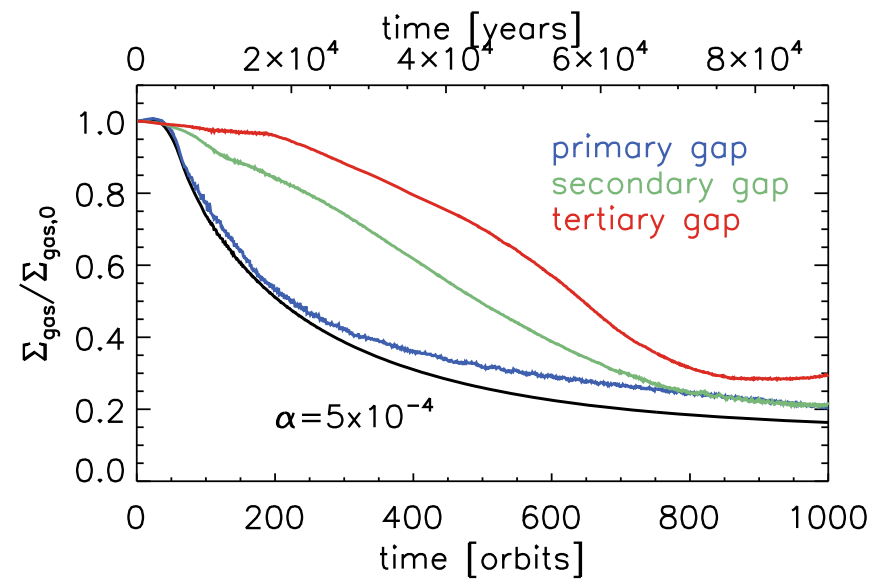

Figure 3. Time evolution of gap depths measured in hydrodynamic simulations, defined as the ratio between the gas surface density at the gap center $\left(\Sigma_{\text {gas }}\right)$ to the initial surface density at that location $\left(\Sigma_{\text {gas }, 0}\right)$. The color curves show the depth of the three gaps for the $\alpha=5 \times 10^{-5}$ model: (blue) the primary gap at $20 \mathrm{au}$, (green) the secondary gap at $\sim 8 \mathrm{au}$, and (red) the tertiary gap at $\sim 4$ au. The black curve shows the depth of the primary gap (at $20 \mathrm{au}$ ) for the $\alpha=5 \times 10^{-4}$ model.

addition, it opens two gaps in the inner disk at about 8 and $4 \mathrm{au}$, where the secondary and tertiary spiral arms shock disk gas (Bae et al. 2017). As a result, three pressure bumps develop in the disk: at $\sim 6$ au between the tertiary and secondary gaps, at $\sim 12$ au between the secondary and primary gaps, and at $\sim 33$ au beyond the primary gap. When $\alpha=5 \times 10^{-4}$ is used, on the other hand, the planet opens only the primary gap around its orbit. This is because the angular momentum transport induced by the secondary and tertiary spiral shocks does not exceed that induced by viscous transport of the disk.

The gap depth in both models reaches a quasi-steady state by 1000 planetary orbital times as shown in Figure 3. In the model with $\alpha=5 \times 10^{-4}$ the primary gap depth is consistent with the analytic estimates and the depths obtained in numerical simulations from the literature (e.g., Duffell \& MacFadyen 2013; Fung et al. 2014; Kanagawa et al. 2015). In the case where $\alpha=5 \times 10^{-5}$ is assumed, the primary gap is expected to further deepen over a fraction of the gas viscous timescale (e.g., Fung et al. 2014). However, the drift timescale of grains determining the disk morphology in the (sub)millimeter continuum is much shorter than the gas viscous timescale and, as a result, these grains are drifted toward the adjacent pressure bumps well before the gas viscous timescale is passed, as we will show below.

Figure 4 presents results from the grain evolution calculations after 0.3 Myr. In Model 1, the maximum grain size is set mainly by the radial drift because the fragmentation is inefficient. The relative velocity between equal-sized grains due to turbulence can be expressed as $\sqrt{3 \alpha_{\text {turb }} /(S t+1 / S t)} c_{s}$ (e.g., Cuzzi \& Weidenschilling 2006; Ormel \& Cuzzi 2017). With a small $\alpha_{\text {turb }}=5 \times 10^{-5}$ the maximum relative velocity, which occurs for $S t=1$ grains, is smaller than the assumed fragmentation velocity $\left(v_{\text {frag }}=10 \mathrm{~m} \mathrm{~s}^{-1}\right.$ ) except for inner few astronomical units of the disk. Grains therefore grow with negligible fragmentation until they radially drift toward the pressure bumps. At the pressure bumps, however, the drift timescale is infinitely long and the radial drift cannot limit the growth of grains. Within pressure bumps in a disk having sufficiently low turbulence, grains can thus break both fragmentation and radial drift limits and grow beyond a meter in size.
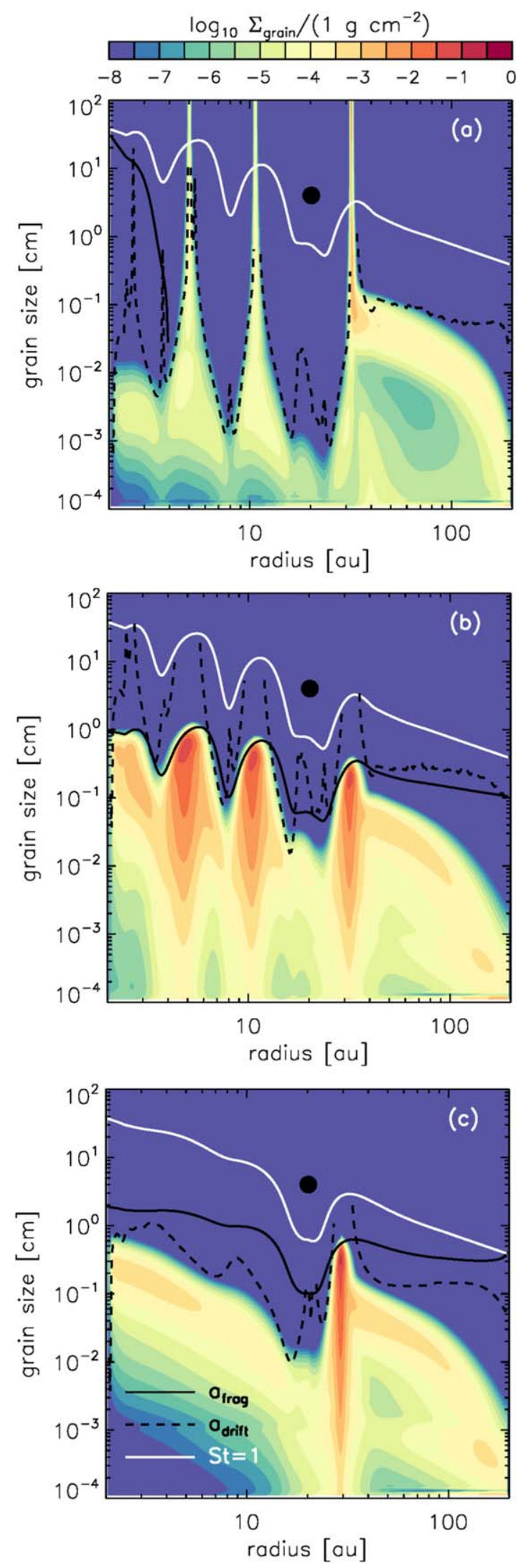

Figure 4. Color contours showing the radial distribution of grains with various sizes for (a) Model 1, (b) Model 2, and (c) Model 3. Each horizontal line presents the surface density of the grain $\left(\Sigma_{\text {grain }}\right)$ with the size shown on the $y$-axis. The white curves show the grain size with Stokes number of unity, while black solid and dashed curves present maximum grain sizes under the fragmentation limit $\left(a_{\text {frag }}\right)$ and the radial drift limit $\left(a_{\text {drift }}\right)$, respectively, calculated using Equations (1) and (3). 
Another noticeable feature in this model is that the widths of the dust rings are much narrower than the widths of the gas pressure bumps due to a runaway radial drift (one may compare the width of dust rings in Model 1 with that in Model 2). Since the radial drift dominates the grain distribution, grains drift toward the adjacent pressure peak as they grow. The gaps between the pressure peaks lose grains, and this loss leads to an increasingly more efficient radial drift because the growth timescale of grains increases with less grain abundance $\left(\tau_{\text {grow }} \propto \Sigma_{\text {grain }}^{-1}\right.$; Birnstiel et al. 2012). This allows more time for grains to be drifted toward pressure bumps so the drift barrier gradually extends to smaller grain sizes over time. As a result of this runaway radial drift, grains are collected in a radial region whose width is much narrower than the width of the gas pressure bump.

When $\alpha_{\text {turb }}$ is increased to $10^{-3}$, the fragmentation limits the growth of grains. Due to the efficient fragmentation, the maximum grain size in the pressure bumps remains at about a few millimeters to a centimeter. Also, as grains fragment before they grow to experience rapid radial drift, the widths of grain rings remain similar to the widths of pressure bumps. The widths of grain rings in this efficient fragmentation model are therefore much wider than in Model 1.

In Model 3, the maximum size of grains in the inner disk is determined by the radial drift. In the pressure bump at the outer gap edge the maximum size of grains is first set by the radial drift, but when grains further drift toward the pressure peak fragmentation determines the maximum size.

We found in all three models that the grain size distribution in pressure bumps follows a power-law distribution with a slope close to the nominal value of $p=-3.5$, where $d n / d a \propto a^{p}$. However, as can be inferred from Figure 4 the amount of grains dominating the continuum opacity at millimeter wavelengths is very different among the models, but also among different radial locations within each model. For instance, due to the rapid grain growth, millimeter-sized grains are much less abundant in Model 1 than in the other models. Because the total grain mass in Model 1 is dominated by the large grains with sizes of $\gg \mathrm{mm}$, which do not contribute to opacity at millimeter wavelengths, the opacity within the bumps is very small $\left(<0.1 \mathrm{~cm}^{2} \mathrm{~g}^{-1}\right.$ at $1.3 \mathrm{~mm}$ ) as shown in Figure 5.

In all three models, the continuum opacity changes significantly across the disk. We thus caution that using a single opacity value when converting the observed continuum flux to the disk's dust mass or dust surface density profile may lead to inaccurate results.

\section{Disk Morphology in Millimeter Continuum}

In order to examine the morphology of the model disks in millimeter continuum, we make synthesized images using the grain distribution presented in Figure 4. We place disks at $100 \mathrm{pc}$, with their poles aligned along the line of sight (i.e., $0^{\circ}$ inclination). We use CASA version 5.1.2 $2^{4}$ to produce synthesized continuum emission. Four different antenna configurations C43-9, C43-6, C43-5, and C43-4 are considered, with which at $1.3 \mathrm{~mm}(230 \mathrm{GHz})$ we achieve angular resolutions of 0 !" 025 , 0 ." 13,0 ". 24 , and 0 "' 40 , respectively. Thermal noise from the atmosphere and from the antenna receivers is added by setting the thermalnoise option in thesimobserve task to tsys-atm. We use an on-target integration time of 10 minutes for C43-6, C43-5, and C43-4 antenna configurations and of $1 \mathrm{hr}$ for C43-9

\footnotetext{
4 https://casa.nrao.edu/
}
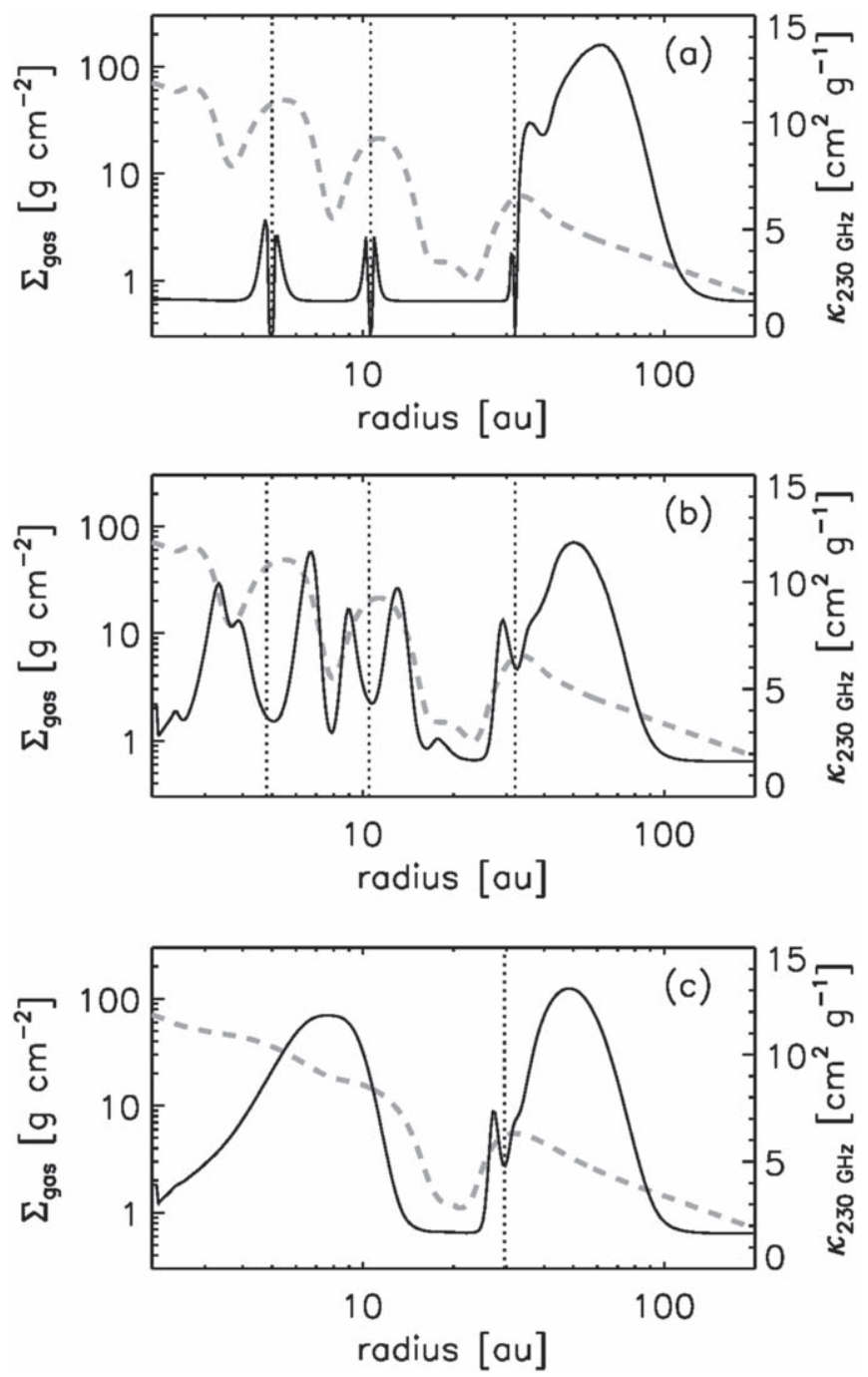

Figure 5. Radial distributions of the gas surface density (gray dashed curves, left $y$-axis) and the continuum opacity at $230 \mathrm{GHz}$ (black solid curves, right $y$-axis) for (a) Model 1, (b) Model 2, and (c) Model 3. The vertical dotted lines indicate the radial locations where the total grain surface density has a local maximum. Note the radial variations of the continuum opacity across the disks. We adopt the optical constants from Ricci et al. (2010) to calculate the grain opacity.

configuration. The latter is chosen to have comparable spatial resolution and integration time to recent ALMA large program on protoplanetary disks (2016.1.00484.L, PI Andrews, S. M.). For the four antenna configurations, we obtain rms noise levels around $0.3,0.1,0.04$, and $0.004 \mathrm{mJy}^{\text {beam }}{ }^{-1}$ for Model 1, and $0.9,0.3,0.1$, and $0.006 \mathrm{mJy}^{-1}$ beam $^{-1}$ for Models 2 and 3 . We assume a uniform dust temperature of $20 \mathrm{~K}$ similar to what is typically assumed in continuum surveys of protoplanetary disks (e.g., Andrews \& Williams 2005; Andrews et al. 2013). Using the gas temperature for the dust temperature increases the absolute flux of disks and makes the inner disk relatively brighter than the outer disk. We also examined the disk morphology using dust evolution calculation outputs at various time epochs, but we found no qualitative difference in the morphology.

Figure 6 presents the synthesized images. The inner two rings have fluxes of 0.003 and $0.006 \mathrm{mJy} \mathrm{beam}^{-1}$ (in the absence of thermal noises), and even with $1 \mathrm{hr}$ of integration the two rings are not detected at the highest resolution in Model 1. This disk would thus appear to be a transition disk 



Model 2 (efficient frag.)
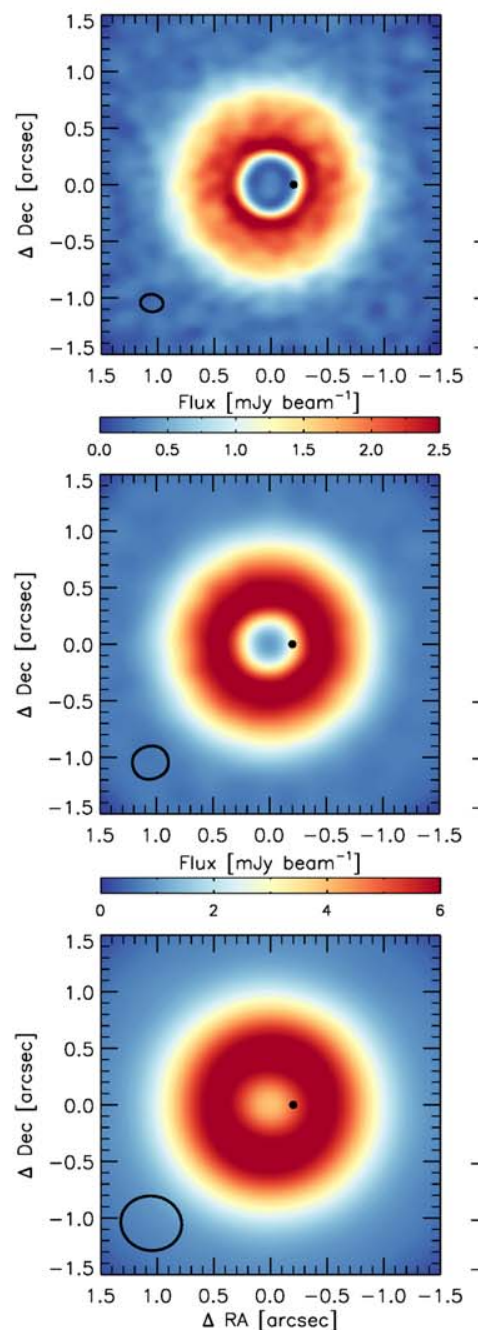
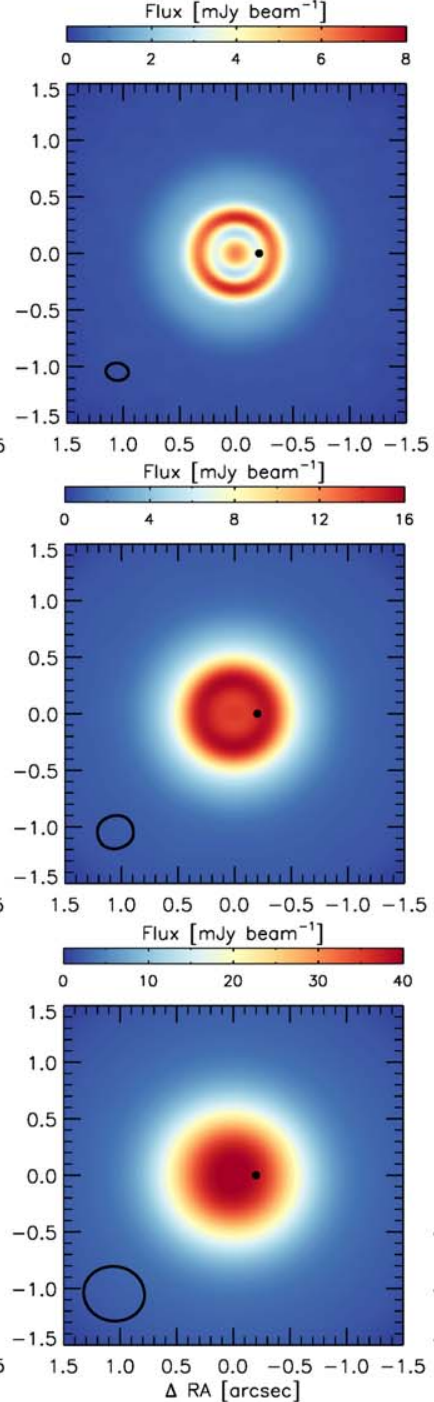

Model 3

(large disk viscosity)
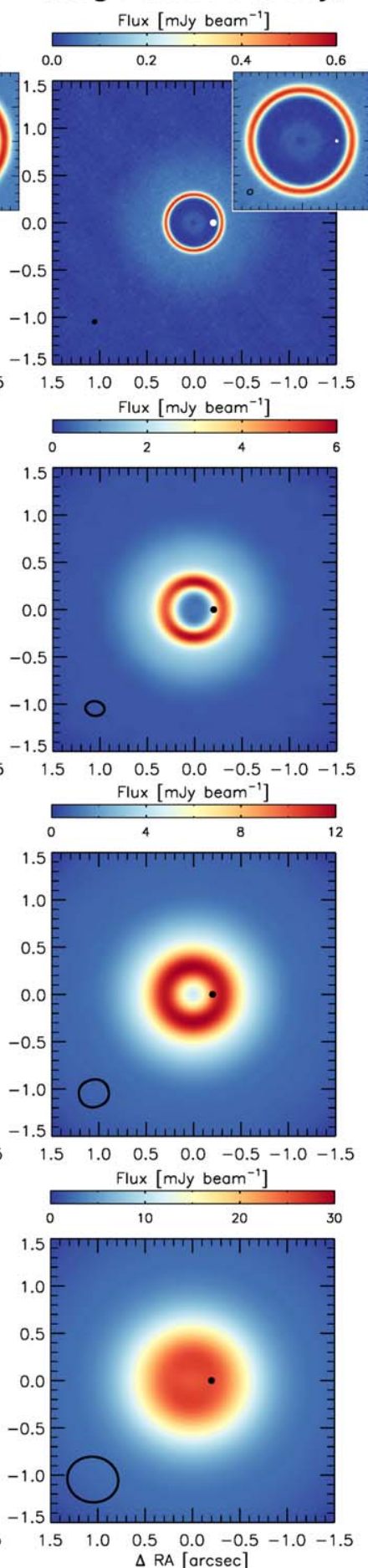

$0 " .025$

resolution

$0 " .13$

resolution

$0 " .24$

resolution

\section{$0 " .40$}

resolution

Figure 6. Simulated millimeter continuum observations of the three models with (first row) $0 . \prime 025$, (second row) 0. .' 13 , (third row) 0 .. 24 , and (fourth row) 0 .! 40 angular resolutions. The beam is shown at the lower left part of each panel with a black contour. The planet is located at 0 !' 2 radial distance from the center (black/ white filled circles). The inset panels in the first row present the inner \pm 0 ". 4 of the disks. The dotted circles in the inset of highest resolution Model 1 image indicate the locations of the inner two rings.

with an inner cavity at all spatial resolutions. The rings have lower fluxes by a factor of $\sim 10$ compared with other models due to the low small grain abundance (Figure 4; see also de Juan Ovelar et al. 2016). As noted earlier, increasing the dust temperature to gas temperature results in higher absolute flux and reveals the inner two rings at the $10 \sigma$ level using the highest spatial resolution. However, even with the enhanced dust temperature the emission from the inner rings are smeared out at lower spatial resolutions, and the disk appears to have an inner cavity.

All three rings in Model 2 contain sufficient amounts of small grains contributing to millimeter continuum. All the rings 


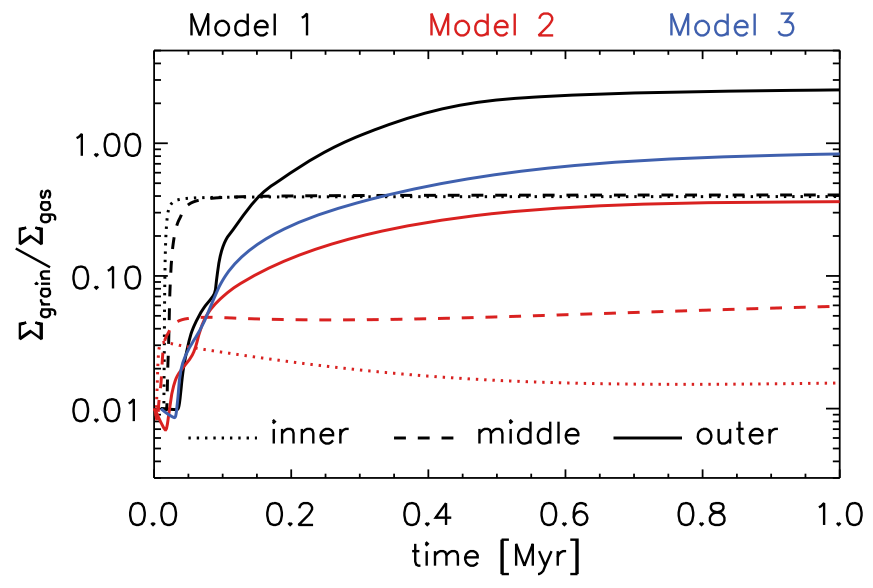

Figure 7. Time evolution of dust-to-gas mass ratio $\Sigma_{\text {grain }} / \Sigma_{\text {gas }}$ at the peak of the dust rings. The black and red curves show the $\Sigma_{\text {grain }} / \Sigma_{\text {gas }}$ values at the peak of dust rings in Models 1 and 2: (dotted) the inner ring at $\sim 5 \mathrm{au}$, (dashed) the middle ring at $\sim 11 \mathrm{au}$, and (solid) the outer ring at $\sim 32$ au. The blue solid curve shows the $\Sigma_{\text {grain }} / \Sigma_{\text {gas }}$ value at the peak of the outer dust ring in Model 3 at $\sim 29$ au

are optically thick and the disk appears to have multiple rings and gaps at the highest spatial resolution. At 0 " 13 resolution, the inner two rings are not resolved and the disk appears to have a single gap around the planet and a central continuum peak. At even lower resolutions, the disk appears to be a transition disk with a shallow inner cavity (with a 0 "' 24 beam) or a full disk without an inner cavity or a gap (with a 0 ". 40 beam).

One important difference between Models 1 and 2 is that the outer ring in Model 1 is narrower than the outer ring in Model 2 as discussed in the previous section. When the maximum grain size within pressure bumps is limited by radial drift (Model 1), this can lead a runaway drift toward the pressure peak, resulting in the formation of a very narrow continuum ring (Figure 4(a)). On the other hand, when the maximum grain size within pressure bumps is limited by fragmentation there is no (or little) drift toward the pressure peak and continuum rings have as broad widths as that of gas pressure bumps (Figure 4(b)). A narrow continuum ring width may therefore imply inefficient fragmentation, presumably hinting at low turbulence in the disk. Alternatively, however, it is possible that a narrow continuum ring width is due to a narrow gas pressure bump width. Having high spatial resolution observations that can resolve continuum rings with multiple beams, together with accurate constraints on the radial gas density profile across rings and gaps (e.g., Teague et al. 2018) and on the disk turbulence (e.g., Flaherty et al. 2015; Teague et al. 2016), will allow us to distinguish the two possibilities.

For Model 3 in which only one pressure bump develops, the disk appears to be a transition disk having an inner cavity until the size of the beam becomes comparable to the size of the inner cavity.

As pressure bumps efficiently trap grains, they can provide a favorable condition to convert grains to planetesimals and/or protoplanets, whose effects are not included in our dust evolution calculations. One such possibility is that the streaming instability is triggered in pressure bumps, converting pebble-sized grains to planetesimals (Youdin \& Goodman 2005; Johansen et al. 2007). In Figure 7, we present the time evolution of the dust-to-gas mass ratio at the peak of dust rings. The dust-to-gas mass ratio reaches $\gtrsim 0.1$ within the first
0.2 Myr of dust evolution, which would be indeed sufficient to trigger the streaming instability (Carrera et al. 2015). In addition, when a disk managed to build a Jupiter-mass planet, it is very likely that there exist planetesimals and/or protoplanets throughout the disk. In such a situation, concentration of grains in pressure bumps can promote the growth of already-existing planetesimals/protoplanets via pebble accretion (Johansen \& Lacerda 2010; Ormel \& Klahr 2010). In fact, more than 20 Earth-masses of solid particles are collected in the outermost pressure bump in Model 1, potentially facilitating the formation of a second-generation (giant) planet there (Lyra et al. 2009; Ronnet et al. 2018; see also Pinilla et al. 2016 for a similar possibility suggested for the outer edge of the dead zone).

When the (sub)millimeter continuum morphology is concerned, a reduction in the amount of grains could decrease the absolute flux of a dust ring. To test the potential effect of the removal of grains via planetesimal-forming/growing processes, we make simulated continuum images after applying a maximum dust-to-gas mass ratio of $\Sigma_{\text {grain }} / \Sigma_{\text {gas }}=0.02$. In practice, at any radius where $\Sigma_{\text {grain }} / \Sigma_{\text {gas }}>0.02$ the grain surface density is reduced (uniformly across different dust sizes) to have $\Sigma_{\text {grain }} / \Sigma_{\text {gas }}=0.02$. We chose this particular value because of the following two reasons. First, $\Sigma_{\text {grain }} / \Sigma_{\text {gas }}=0.02$ is about the dust-to-gas surface density ratio below which the streaming instability is unlikely to operate (Carrera et al. 2015). Second, it is likely that some fraction of small grains would always remain within the rings even in the case of streaming instability and/or when pebble accretion is in action. Numerical simulations show that the conversion rate (in mass) of grains to planetesimals via the streaming instability is less than $50 \%$ (Johansen et al. 2012; Simon et al. 2016). Similarly, the efficiency of pebble accretion onto protoplanets, defined as the ratio between the number of pebbles settled to the protoplanet and the total number of integration of pebble's stochastic equation of motion, is $\lesssim 10 \%$ except at the iceline locations for which the efficiency can be as high as $\sim 50 \%$ (Ormel \& Liu 2018). Furthermore, it is also possible that the collisions among dynamically excited planetesimals in a disk having a giant planet can replenish small grains, as was recently proposed for the multiringed HD 163296 disk (Turrini et al. 2018). So it is reasonable to choose a nonzero $\Sigma_{\text {grain }} / \Sigma_{\text {gas }}$ value.

Figure 8 shows the resulting simulated continuum images. The absolute flux of the continuum rings has been decreased with the reduction in grain amount, but it is found that the overall continuum morphology has not been changed because pressure bumps still have sufficient millimeter-sized grains within the beam. While this experiment shows that the removal of grains through planetesimal-forming/-growing processes would not significantly alter the overall continuum morphology, further investigations on the efficiency of streaming instability and pebble accretion within pressure bumps and considerations of these processes in dust evolution calculations are warranted to conclude this.

\section{Summary and Outlook}

Using hydrodynamic simulations, dust evolution models, and synthetic observations, we showed that a Jupiter-mass planet can produce a diverse protoplanetary disk morphology, including a full disk, a transition disk with an inner cavity, a disk with a single gap and a central continuum peak, and a 

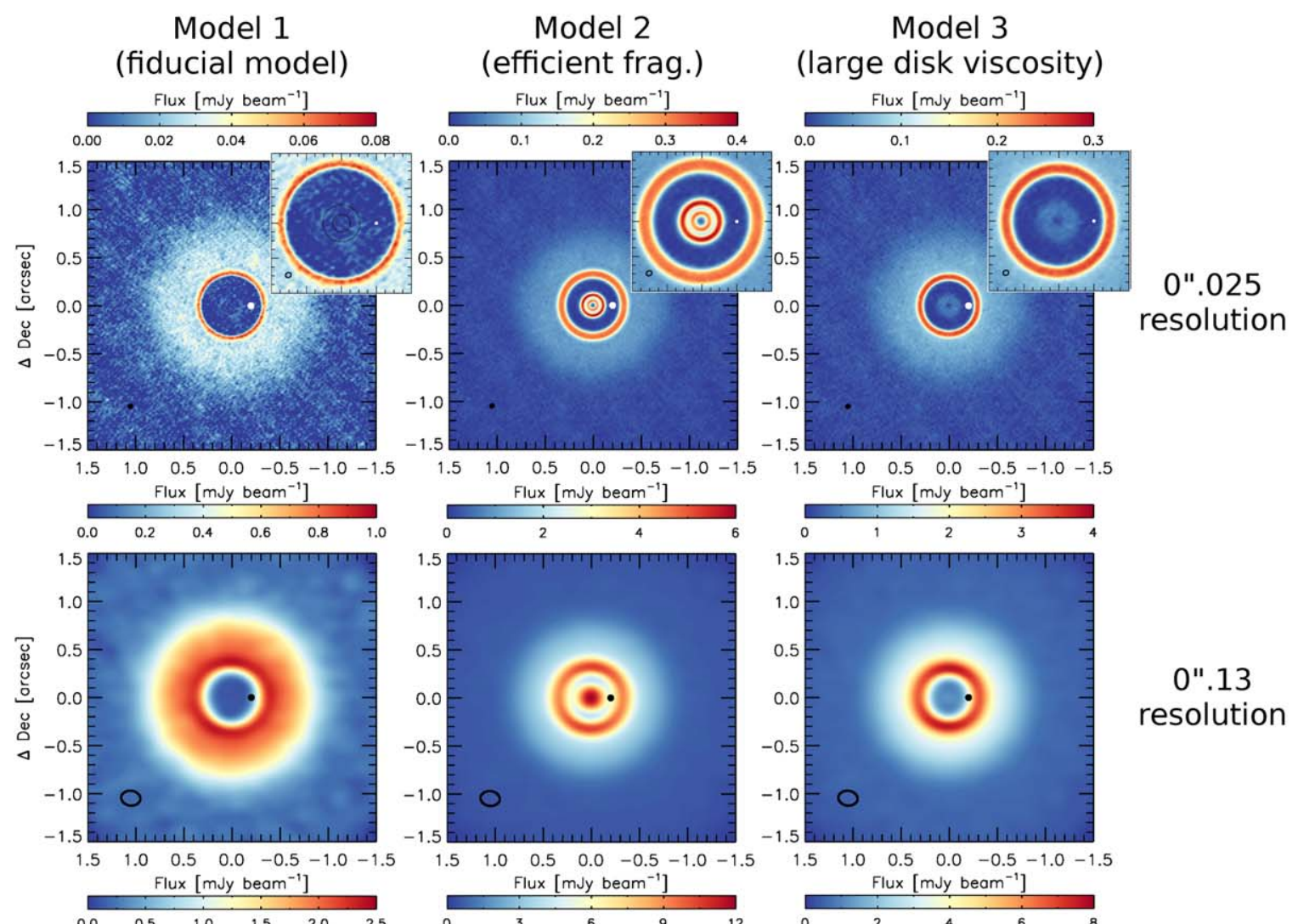

$0 " .13$
resolution
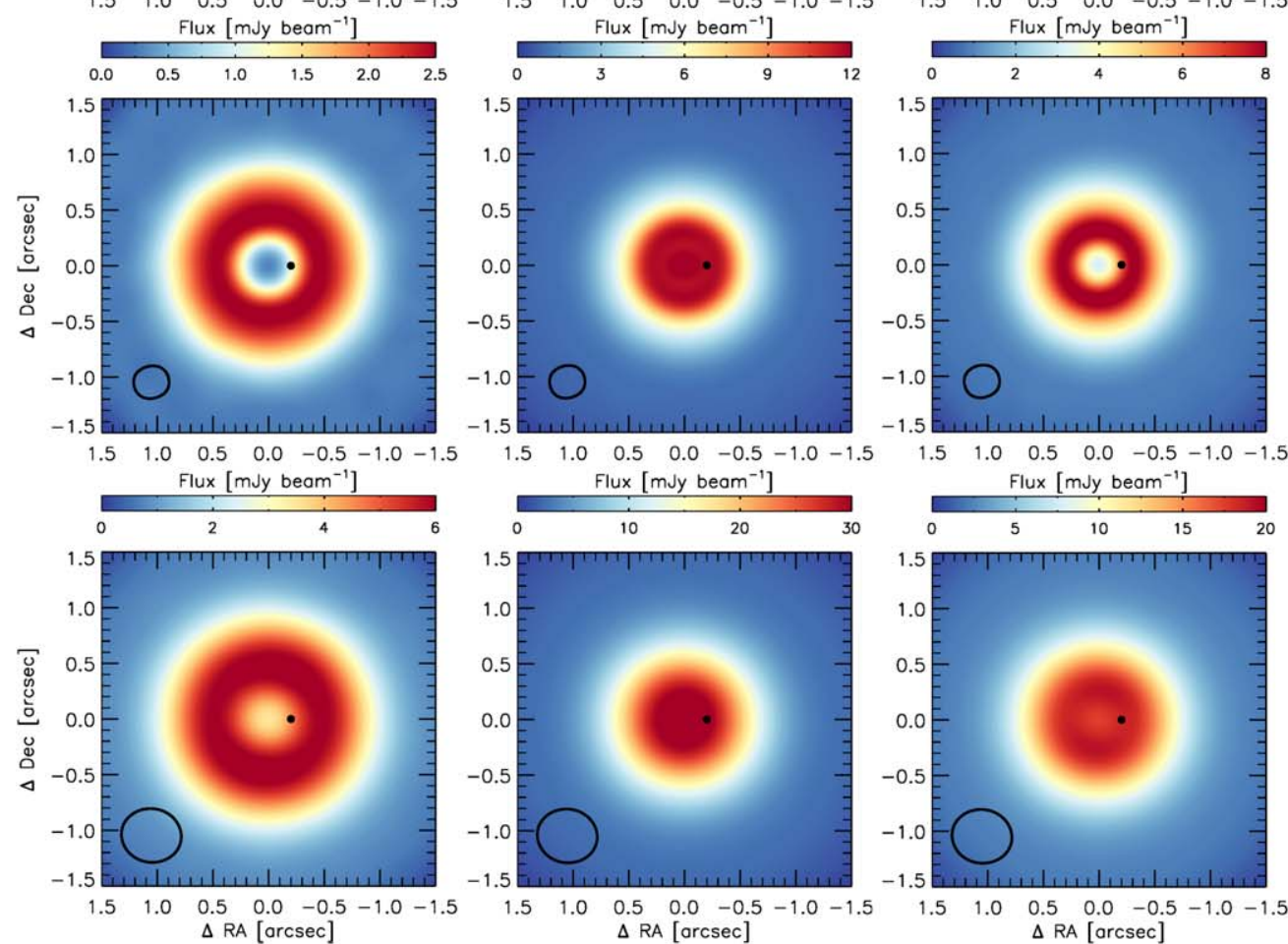

$0 " .24$

resolution

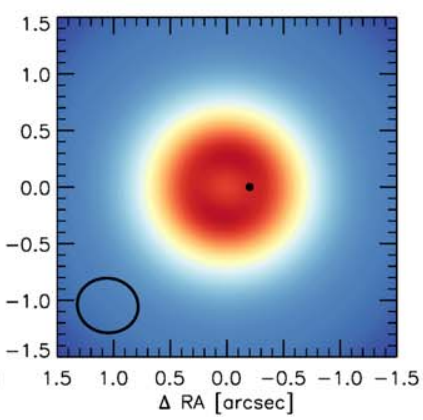

$0 " .40$
resolution

Figure 8. Same as Figure 6, but using the total grain surface at each radius limited to the maximum $2 \%$ of the gas surface density to test potential effects of the removal of small grains through planetesimal formation/growth (see the text). Note that the color bars in Models 2 and 3 have different ranges than Figure 6. The absolute flux of the rings has been decreased with the reduced grain amount, but the overall morphology of the disks is unchanged.

multigapped disk. Such a diversity originates from the level of viscous transport in the disk that determines the number of gaps a planet can open, the grain size distribution set by the radial drift and fragmentation, and the angular resolution used to observe the disk.

Because disks with the same underlying gas distribution can have different millimeter continuum appearance (Model 1 versus 2), complementary molecular line observations that can constrain the disk gas distribution are strongly suggested. In addition, observations with high spatial resolution and sensitivity are necessary to better understand the true nature of protoplanetary disks. Finally, searches for localized signatures of planets, including accretion onto planets (e.g., Sallum et al. 2015), chemical/kinematic signatures in circumstellar disks at 
the vicinity of planets (Cleeves et al. 2015; Pinte et al. 2018), and kinematic/thermal signatures associated with circumplanetary disks (Perez et al. 2015; Zhu et al. 2016, 2018), are highly desired to confirm (or disprove) the presence of planets in the disks with substructures and to differentiate possible causes of rings, gaps, and inner cavities.

As illustrated in Figure 1, the hypothesized gap-opening planets are located in an interesting region of the planet masssemimajor axis plane, for which we do not have counterpart exoplanets discovered with the current exoplanet detection techniques. Future observations with $25+$ meter class telescopes (e.g., E-ELT, GMT, TMT) will offer unprecedented capabilities to directly detect young, self-luminous planets still embedded in protoplanetary disks, allowing us to have critical insights into the formation and evolution of planets.

We thank the anonymous referee for quick and helpful reports that have significantly improved the initial manuscript. J.B. thanks Richard Teague and Ke Zhang for helpful comments on the initial draft of the paper, Scott Gaudi for his help with adding the estimated regions of exoplanet detection to Figure 1, Alycia Weinberger for helpful conversation on future direct imaging observations, and Andrew Youdin for helpful conversation on the streaming instability. J.B. acknowledges support from NASA grant NNX17AE31G and computing resources provided by the NASA High-End Computing (HEC) Program through the NASA Advanced Supercomputing (NAS) Division at Ames Research Center. P.P. acknowledges support by NASA through Hubble Fellowship grant HST-HF2-51380.001-A awarded by the Space Telescope Science Institute, which is operated by the Association of Universities for Research in Astronomy, Inc., for NASA, under contract NAS 5-26555. T.B. acknowledges funding from the European Research Council (ERC) under the European Union's Horizon 2020 research and innovation programme under grant agreement No. 714769.

Software: CASA (McMullin et al. 2007), FARGO3D (Benítez-Llambay \& Masset 2016).

\section{ORCID iDs}

Jaehan Bae (1) https://orcid.org/0000-0001-7258-770X Paola Pinilla (1) https://orcid.org/0000-0001-8764-1780 Tilman Birnstiel (1) https://orcid.org/0000-0002-1899-8783

\section{References}

ALMA Partnership, Brogan, C. L., Pérez, L. M., et al. 2015, ApJL, 808, L3 Andrews, S. M., Rosenfeld, K. A., Kraus, A. L., \& Wilner, D. J. 2013, ApJ, 771,129

Andrews, S. M., \& Williams, J. P. 2005, ApJ, 631, 1134

Andrews, S. M., Wilner, D. J., Zhu, Z., et al. 2016, ApJL, 820, L40

Avenhaus, H., Quanz, S. P., Garufi, A., et al. 2018, ApJ, 863, 44

Bae, J., \& Zhu, Z. 2018a, ApJ, 859, 118

Bae, J., \& Zhu, Z. 2018b, ApJ, 859, 119

Bae, J., Zhu, Z., \& Hartmann, L. 2016, ApJ, 819, 134

Bae, J., Zhu, Z., \& Hartmann, L. 2017, ApJ, 850, 201

Benítez-Llambay, P., \& Masset, F. 2016, ApJS, 223, 11

Birnstiel, T., Andrews, S. M., Pinilla, P., \& Kama, M. 2015, ApJL, 813, L14
Birnstiel, T., Dullemond, C. P., \& Brauer, F. 2010, A\&A, 513, A79 Birnstiel, T., Klahr, H., \& Ercolano, B. 2012, A\&A, 539, A148

Carrera, D., Johansen, A., \& Davies, M. B. 2015, A\&A, 579, A43

Cleeves, L. I., Bergin, E. A., \& Harries, T. J. 2015, ApJ, 807, 2

Currie, T., Ryan, C., \& Brittain, S. 2015, ApJL, 814, L27

Cuzzi, J. N., \& Weidenschilling, S. J. 2006, in Meteorites and the Early Solar System II, ed. D. S. Lauretta \& H. Y. McSween, Jr. (Tucson, AZ: Univ. Arizona Press), 353

de Juan Ovelar, M., Pinilla, P., Min, M., Dominik, C., \& Birnstiel, T. 2016, MNRAS, 459, L85

Dipierro, G., Ricci, L., Peréz, L., et al. 2018, MNRAS, 475, 5296

Dong, R., \& Fung, J. 2017a, ApJ, 835, 146

Dong, R., \& Fung, J. 2017b, ApJ, 835, 38

Dong, R., Fung, J., \& Chiang, E. 2016, ApJ, 826, 75

Dong, R., Li, S., Chiang, E., \& Li, H. 2017, ApJ, 843, 127

Dong, R., Zhu, Z., Rafikov, R. R., \& Stone, J. M. 2015, ApJL, 809, L5

Duffell, P. C., \& MacFadyen, A. I. 2013, ApJ, 769, 41

Dullemond, C. P., \& Penzlin, A. B. T. 2018, A\&A, 609, A50

Fedele, D., Tazzari, M., Booth, R., et al. 2018, A\&A, 610, A24

Flaherty, K. M., Hughes, A. M., Rosenfeld, K. A., et al. 2015, ApJ, 813, 99

Flock, M., Nelson, R. P., Turner, N. J., et al. 2017, ApJ, 850, 131

Follette, K. B., Rameau, J., Dong, R., et al. 2017, AJ, 153, 264

Fung, J., Shi, J.-M., \& Chiang, E. 2014, ApJ, 782, 88

Gaudi, B. S. 2012, ARA\&A, 50, 411

Hashimoto, J., Tamura, M., Muto, T., et al. 2011, ApJL, 729, L17

Isella, A., Guidi, G., Testi, L., et al. 2016, PhRvL, 117, 251101

Jin, S., Li, S., Isella, A., Li, H., \& Ji, J. 2016, ApJ, 818, 76

Johansen, A., \& Lacerda, P. 2010, MNRAS, 404, 475

Johansen, A., Oishi, J. S., Mac Low, M.-M., et al. 2007, Natur, 448, 1022

Johansen, A., Youdin, A., \& Klahr, H. 2009, ApJ, 697, 1269

Johansen, A., Youdin, A. N., \& Lithwick, Y. 2012, A\&A, 537, A125

Kanagawa, K. D., Tanaka, H., Muto, T., et al. 2015, MNRAS, 448, 994

Keppler, M., Benisty, M., Müller, A., et al. 2018, A\&A, in press (arXiv:1806. 11568)

Lin, D. N. C., \& Papaloizou, J. 1980, MNRAS, 191, 37

Lyra, W., Johansen, A., Klahr, H., \& Piskunov, N. 2009, A\&A, 493, 1125

Masset, F. 2000, A\&AS, 141, 165

McMullin, J. P., Waters, B., Schiebel, D., et al. 2007, in ASP Conf. Ser. 376, Astronomical Data Analysis Software and Systems XVI, ed. R. A. Shaw, F. Hill, \& D. J. Bell (San Francisco, CA: ASP), 127

Meru, F., Juhász, A., Ilee, J. D., et al. 2017, ApJL, 839, L24

Okuzumi, S., Momose, M., Sirono, S.-i., Kobayashi, H., \& Tanaka, H. 2016, ApJ, 821, 82

Ormel, C. W., \& Cuzzi, J. N. 2017, A\&A, 466, 413

Ormel, C. W., \& Klahr, H. H. 2010, A\&A, 520, A43

Ormel, C. W., \& Liu, B. 2018, A\&A, 615, A178

Perez, S., Dunhill, A., Casassus, S., et al. 2015, ApJL, 811, L5

Pinilla, P., Birnstiel, T., Ricci, L., et al. 2012, A\&A, 538, A114

Pinilla, P., Flock, M., de Juan Ovelar, M., \& Birnstiel, T. 2016, A\&A, 596, A81

Pinte, C., Price, D. J., Menard, S., et al. 2018, ApJL, 860, L13

Quanz, S. P., Amara, A., \& Meyer, M. R. 2013, ApJL, 766, L1

Ricci, L., Testi, L., Natta, A., et al. 2010, A\&A, 512, A15

Ronnet, T., Mousis, O., Crida, A., et al. 2018, AJ, 155, 224

Sallum, S., Follette, K. B., Eisner, J. A., et al. 2015, Natur, 527, 342

Shakura, N. I., \& Sunyaev, R. A. 1973, A\&A, 24, 337

Sheehan, P. D., \& Eisner, J. A. 2018, ApJ, 857, 18

Simon, J. B., Armitage, P. J., Li, R., \& Youdin, A. N. 2016, ApJ, 822, 55

Tang, Y.-W., Guilloteau, S., Dutrey, A., et al. 2017, ApJ, 840, 32

Takahashi, S. Z., \& Inutsuka, S.-I. 2014, ApJ, 794, 55

Teague, R., Bae, J., Bergin, E. A., Birnstiel, T., \& Foreman-Mackey, D. 2018, ApJL, 860, L12

Teague, R., Guilloteau, S., Semenov, D., et al. 2016, A\&A, 592, A49

Tsukagoshi, T., Nomura, H., Muto, T., et al. 2016, ApJL, 829, L35

Turrini, D., Marzari, F., Polychroni, D., \& Testi, L. 2018, arXiv:1802.04361

Youdin, A. N., \& Goodman, J. 2005, ApJ, 620, 459

Zhang, K., Bergin, E. A., \& Blake, G. A. 2016, ApJL, 818, L16

Zhang, K., Blake, G. A., \& Bergin, E. A. 2015, ApJL, 806, L7

Zhu, Z., Andrews, S. M., \& Isella, A. 2018, MNRAS, 479, 1850

Zhu, Z., Ju, W., \& Stone, J. M. 2016, ApJ, 832, 193 